\title{
De la Rémunération du Personnel dans les Structures Professionnelles des Eglises à Bukavu
}

\author{
Habamungu Bashwira, \\ Chef de Travaux à l'UOB, Département de Sociologie, \\ République Démocratique du Congo \\ Lokanga Otikeke,
} Professeur à l'Université de Kisangani, République Démocratique du Congo

Doi:10.19044/esj.2020.v16n10p311 URL:http://dx.doi.org/10.19044/esj.2020.v16n10p311

\section{Résumé}

Le présent article porte sur la rémunération dans les structures professionnelles des Eglises à Bukavu. L'étude vise à (1) déterminer les masses salariales allouées aux agents par les structures professionnelles des Eglises ; (2) jauger le degré de satisfaction et de motivation des agents face à la rémunération qu'ils reçoivent et (3) à identifier les lignes des dépenses couvertes par ces salaires dans la Ville de Bukavu. Pour la collecte des données, 401 sujets issus de 42 structures professionnelles des Eglises de Bukavu ont été enquêtés à l'aide de l'outil questionnaire. La méthode structuro-fonctionnelle, l'analyse de contenu et le test chi carré ont soutenu le dépouillement et le traitement des données. Il ressort des résultats de la recherche que le salaire est un aspect important dans le fonctionnement harmonieux de toute structure professionnelle. Les masses salariales allouées aux agents varient selon les tailles, les productivités, l'accessibilité à la main d'œuvre et la bonne volonté des structures professionnelles des Eglises. Le salaire alloué aux agents oscille entre 0 et 2000 dollars par mois et par individu. Ce salaire ne suffit pas pour couvrir les besoins d'épargne et d'investissement. En dépit, de sa faiblesse à satisfaire aux besoins des agents, la rémunération est relativement bien payée par les structures professionnelles des Eglises protestantes plutôt que celles de l'Eglise catholique. Les agents sont ainsi démotivés et restent à la recherche permanente d'un travail qui procurerait la bonne rémunération.

Mots clés : Emploi, Rémunération, Salaire, Eglises, motivation 


\title{
Staff Remuneration in the Professional Structures of Churches in Bukavu
}

\author{
Habamungu Bashwira, \\ Chef de Travaux à l'UOB, Département de Sociologie, \\ République Démocratique du Congo \\ Lokanga Otikeke,
}

Professeur à l'Université de Kisangani, République Démocratique du Congo

\begin{abstract}
This paper focuses on determining the amount of wages allocated to employees in the Church organizations and enterprises. It assesses the extent to which employees are satisfied and motivated based on the wages they are paid, and it also examines how Church employees allocate their wages to meet the needs of their households. Data was collected in Bukavu through a selfadministered questionnaire on a sample of 401 Church employees. The data was processed and analyzed in SPSS and Excel through the structural functional method, together with content analysis and Chi-Square test. The findings indicate that salary is an important driver of smooth relationships and it is very important for the functioning of any Church organization or enterprise. The total amount of wages allocated to employees varies among Church organizations and enterprises. This is dependent on the organizational size, productivity, location of majority of the workforce, and the policy of the Church enterprise. On average, the salary per individual worker ranges from 0 to 2,000 dollars per month. The salary is not enough to cover savings and investment needs of employees, rather it only serves for subsistence needs such as food, school fees of children, and rent. The remuneration is higher for employees who work for protestant organizations and enterprises compared to Catholic Church. The employees therefore are unsatisfied, not motivated, and are in a continuous search for a better job.
\end{abstract}

Keywords: Employment, remuneration, salary, motivation, churches

\section{Introduction}

La politique de la rémunération représente un axe important de la politique de gestion des ressources humaines de l'entreprise en ce sens qu'elle exige la conciliation, au même moment, des considérations économiques 
(recherche de performance et d'efficacité) et des considérations sociales (reconnaissance du capital, objectif d'équité, motivation).

Toute politique de rémunération poursuit les missions sociale, économique et politique. Du point de vue social, la politique salariale vise la répartition équitable des revenus du travailleur et sa famille, la valorisation de la personnalité du travailleur. Du point de vue économique, les objectifs de la politique salariale sont l'accroissement du pouvoir d'achat des ménages de manière à stimuler la productivité intérieure, l'amélioration de la productivité du rendement, la mise en valeur d'une partie de salaire de manière à constituer l'épargne nationale, nivelée par des épargnes individuelles. S'agissant de l'aspect politique, la répartition équitable des salaires entre les hauts cadres et la base occasionne souvent la stabilité politique dans l'entreprise.

La rémunération est à la fois un facteur d'équilibre social pour les salariés et un coût pour l'Entreprise. «Jusqu'au début du XXe siècle le paiement du travail se faisait essentiellement sous forme de gages puis la notion de salaire apparait progressivement. Dans 1'entreprise taylorienne est instauré le salaire au rendement avec la fixation d'une rémunération à la pièce afin d'obtenir une meilleure performance. Après la seconde guerre mondiale, le salaire à la pièce est remplacé par un salaire fixe et collectif. Le salaire de base fixe est éventuellement augmenté de primes ». (Guillot-Soulez, 2010).

Si la rémunération est un coût pour les entrepreneurs, elle fait souvent l'objet des disputes dans le processus de sa libération. Lorsqu'elle est bien libérée, elle crée la confiance entre les partenaires au travail c'est-à-dire l'employeur et l'employé. Par contre, lorsqu'elle n'est pas bien libérée ou libérée en partie ou avec retard, elle crée des frustrations et les conflits professionnels s'en suivent. Guidés par le principe du plus grand profit, les entrepreneurs cherchent à maintenir le salaire le plus bas possible pour le travail exécuté par les ouvriers. Friedmann et Navile (1972) notent à ce sujet que l'homme tout entier est modelé par l'activité pratique, son travail oriente sa façon d'être, sa conduite, ses comportements.

Les conséquences de l'insatisfaction envers la rémunération sont multiples. Werther et al. (1990) relèvent les conséquences ci-après : la démission, la baisse du rendement, l'augmentation de griefs, l'absentéisme, l'insatisfaction au travail, etc. « La rémunération trop généreuse est source de problèmes. Un coût de rémunération élevé limite la compétitivité de l'organisation et sa capacité à offrir des emplois. Il faut donc un équilibre entre la satisfaction envers la rémunération et la compétitivité de l'organisation ». (Mokakando, 2012).

Le problème de rémunération se pose avec acuité dans la ville de Bukavu. Le constat empirique laisse voir un écart criant entre la rémunération du secteur religieux et celle d'autres secteurs professionnels. C'est pour cette 
raison que le Pape Jean Paul II « préconise une juste rémunération du travail de l'adulte chargé de famille. » (Le Pape Jean Paul II, 1981).

Le présent article propose un essai de compréhension de trois dimensions de la rémunération dans les secteurs professionnels promus par les Eglises dans la Ville de Bukavu. La première dimension est celle des taux et du sens de la rémunération dans la Ville de Bukavu. La seconde dimension est celle de l'étude comparative de la rémunération chez les catholiques et chez les protestants. Enfin, la troisième dimension de recherche consistera à réexaminer, les rapports entre la rémunération, le degré de satisfaction des besoins et la motivation au travail confessionnel par les employés, en les situant dans une perspective d'équité ventée par les discours religieux.

Il s'agit globalement d'analyser l'ensemble de dispositions arrêtées au sein des confessions religieuses pour rémunérer les employés en tenant compte de leur profil et participation à la production ou au fonctionnement harmonieux des entreprises qui les utilisent.

Eu égard à cela, cette recherche part des questionnements ci-après :

1. Quels sont les facteurs déterminants les niveaux de salaires alloués au personnel dans les structures professionnelles religieuses à Bukavu ?

2. Quelles sont les particularités constatées chez les catholiques et chez les protestants dans leurs politiques de rémunération du personnel à Bukavu?

3. Comment les masses salariales sont-elles perçues par les agents des confessions religieuses à Bukavu par rapport à la satisfaction de leurs besoins quotidiens?

Pour répondre à ces questionnements, les hypothèses suivantes ont été émises :

$1^{\circ}$ ) Le niveau de salaire dans une structure professionnelle religieuse est fonction du coût de vie, de la pénurie d'offre de travail, de la productivité, du pouvoir de négociation des travailleurs organisés en syndicat ou en association. Le niveau général de salaire n’est que la moyenne de salaire.

$2^{\circ}$ ) Le salaire diffère significativement selon les confessions religieuses. La moyenne salariale octroyée par les services des confessions protestantes serait supérieure à celle qu'on donne au niveau de la confession catholique. Les protestants seraient plus libéraux et respectueux des droits de travailleurs plus que les catholiques.

$3^{\circ}$ ) La satisfaction salariale serait déplorable chez les agents des structures professionnels des Eglises à Bukavu. Les structures professionnelles ne sont pas bien appréciées par leurs agents en matière de paiement de salaire. Le salaire alloué aux agents par les services 
promus par les Eglises ne permet pas à ces derniers de répondre à leurs besoins élémentaires.

\section{Champ d'etude}

La Ville de Bukavu est située à l'Est de la République Démocratique du Congo à 28 51' de longitude Est et à une altitude moyenne de $1600 \mathrm{~m}$. Elle est la ville la plus élevée du pays. Chef-lieu de l'ancienne Province du Kivu, Bukavu a été créé par l'ordonnance N²2/1396 du 29/06/1958 qui en fixe les limites. La Ville de Bukavu est devenue la capitale de la Province du SudKivu, par l'ordonnance-loi N88-031 du 20 juillet 1988 portant découpage territorial divisant l'ancienne Province du Kivu en trois provinces distinctes : celle du Maniema, celle du Nord-Kivu et celle du Sud-Kivu. (Pilo \& Rugambwa, 2005)

Cette Ville est limitée au Nord par le Lac Kivu, au Sud et à l'Ouest par le Territoire de Kabare et à l'Est par la rivière Ruzizi qui forme une frontière naturelle avec le Rwanda. La Ville de Bukavu compte 1.184.955 habitants nationaux et étrangers en 2017. Ce nombre d'habitants est installé sur une superficie de 44,90 $\mathrm{Km}^{2}$.

Sur le plan professionnel, Cishunguluka (2017) note qu'il y existe dans la Ville de Bukavu des travailleurs et des chômeurs, des actifs et des passifs sociaux. C'est une ville où les études et, de surcroît, les diplômes sont perçus comme la clé de voute pour l'accès à l'emploi. Les études y sont considérées à la fois comme une arme d'intégration socioprofessionnelle et un prestige social. Sur le plan religieux, la ville de Bukavu est majoritairement chrétienne. Cette ville renferme beaucoup de confessions religieuses à savoir catholique, protestante, anglicane, Kimbanguiste, Musulmane, témoins de Jéhovah, etc. qui créent et gèrent des emplois.

De nombreuses Eglises, surtout celles dites de réveil, fonctionnent sans se doter de la personnalité juridique pendant que 1 'article 47 , alinéa $2^{\text {ème }}$ de la loi $n^{\circ} 004 / 2001$ du 20 juillet 2001 dispose que «Nul ne peut percevoir des dons, présents, legs ou aumônes au nom d'une association confessionnelle n'ayant pas la personnalité juridique ou l'autorisation provisoire de fonctionnement (La loi n004/2001 du 20 juillet 2001).

Il est vrai que ces Eglises s'appuient sur l'article 22 de la constitution actuelle de la République Démocratique du Congo qui stipule que «Toute personne a droit à la liberté de pensée, de conscience et de religion. Toute personne a le droit de manifester sa religion ou ses convictions, seule ou en groupe, tant en public qu'en privé, par le culte, l'enseignement, les pratiques, l'accomplissement des rites et l'état de vie religieuse sous réserve du respect de la loi, de l'ordre public, des bonnes mœurs et des droits d'autrui. (La constitution de la RDC du 18 février 2006). 
Il est difficile de dégager le nombre exact de membres des Eglises de réveil au Congo, en général, et dans la Ville de Bukavu, en particulier, pour des raisons multiples (Lecuyer et al., 2003). Leurs membres sont mobiles et souvent adeptes de plusieurs Eglises à la fois. Le constat empirique montre que certains membres sont à la recherche parfois de l'argent, des hommes et/ou des femmes, mais rarement de Dieu. Pour ce genre de fidèles, l'Eglise offre un bon cadre pour solliciter l'emploi ou un avantage auquel on n'aurait pas d'accès sans adhérer à un mouvement religieux.

Les confessions religieuses opèrent généralement dans six secteurs principaux de la vie sociale. Il s'agit respectivement des secteurs de la santé, de l'éducation, paroissial, culturel et de l'ESU, de l'agro-pastoral, de l'économie solidaire et du développement.

Le secteur de la santé : l'Eglise catholique, la $5^{\text {ème }}$ CELPA et la $8^{\text {ème }}$ CEPAC ont créé des structures sanitaires qui recrutent un personnel non négligeable à Bukavu. Ces structures sont en majorité bien équipées et standardisées aux normes mondiales d'organisation et de fonctionnement. Elles gèrent les grands hôpitaux et centres de santé de la Ville de Bukavu.

A titre d'exemple, l'Eglise catholique gère 2 hôpitaux (l'hôpital provincial général de référence de Bukavu, l'hôpital Dr RAU de Ciriri), 1 Pharmacie (la pharmacie diocésaine) et 14 Centres de Santé (Lumu, Mushekere, Uhaki, Ciriri, Maendeleo, Nyamulagira, Binamé, Funu, Uzima, Maria, Charles Mbogha/labotte, Muhungu, Gihamba, Chai) ainsi que 5 mutuelles de Santé (Ibanda, Kadutu, Bagira, Ciriri et la Mutuelle des étudiants), 1 CEDER (Centrale de Distribution Régionale des médicaments) ... La $8^{\text {ème }}$ CEPAC gère, pour sa part, 1 Hôpital Général de référence de Panzi, 1 Centre hospitalier de Cahi ; le Grand Dépôt pharmaceutique, 9 Centres de santé (Ihemba/CEPAC, Nyengo, Kabuye, Kamagema, Kalagane, Nyengo, Buholo II, Mulambula et Muhungu) et 4 dispensaires. La $5^{\text {ème }}$ CELPA compte à son actif 1 centre hospitalier (Centre hospitalier de la $5^{\text {ème }}$ CELPA), 7 Centres de santé (Muhungu, Bagira, Cifuma, Buholo, Kadutu, Panzi et Igoki) et 6 dispensaires.

Le secteur de l'éducation : placé en deuxième position des secteurs pourvoyeurs des emplois confessionnels à Bukavu, le secteur de l'éducation formelle et non formelle concerne les niveaux maternel, primaire, secondaire et professionnel, ainsi que l'alphabétisation des adultes et la remise à niveau. Au-delà des opportunités d'emplois qu'offre le système scolaire, il joue également la fonction d'encadrement spirituel des chrétiens et de développement de la société.

Le secteur du mouvement associatif et du développement : Sous ce secteur, nous regroupons tous les emplois relevant des organisations non gouvernementales internationales ou locales du ressort confessionnel. Il s'agit des structures professionnelles de l'Eglise catholique comme la Caritas- 
développement, AAP (Agence d'Achat de Performances), Louvaindéveloppement, Misereor, Cordaid, Fondation Mamore, le PEDER, Centre Tupendane/Giovani, Centre Mwanga, Centre Ek'abana, le Comité Anti Bwaki, Commission Justice et Paix, le Groupe Jérémie, la CODILUSI (Commission Diocésaine de Lutte contre le SIDA), PAF (Programme d'Action Famille), REMISACO (Réseau des Mutuelles de santé du Congo), Supervision médicale. Pour ce qui est de la $8{ }^{\text {ème }}$ CEPAC, nous avons identifié le PMU inter life, ZOA, Héritiers de la Justice, WOLD VISION, RIO (Réseau d'Innovation Organisationnelle), Fondation Panzi, Equipe Mobile Médicale (constituée des médecins, anesthésistes, infirmiers effectuant des missions dans toute la République pour soigner les femmes violées, Mission de construction des hôpitaux dans milieux reculés), Projet Badilika. La $5^{\text {ème }}$ CELPA quant à elle compte à son actif le PRRR (Programme de Rapatriement des Réfugiés Rwandais), Paix pour le Monde (Organisation d'appui aux structures de la $5^{\text {ème }}$ CELPA), le Centre d'Accueil Protestant...

Le secteur culturel et de l'enseignement supérieur et universitaire : ce secteur prend en charge 3.285 postes pour les services socio-culturels, des bibliothèques et librairies. Nous citons à titre illustratif pour l'Eglise catholique l'Humanitas, la bibliothéque des Pères Carmes, KivuPresse, le Centre socio-culturel et spirituel Bakandja, le Centre spirituel Amani, le Centre social Bandari, les maisons d'accueil et procures ;les musées, les salles de spectacles et de fêtes (Concordia, Mgr Kaningu) les terrains de football, les centres de recherche (BEST, CERDAF) les médias( radio Maria malkia wa Amani, les instituts et universités ( institut supérieur de pastorale familiale, le Grand séminaire Notre dame d'Afrique, Université Catholique de Bukavu, Pour la $5^{\text {ème }}$ CELPA, l'imprimerie Shahidi presse, Ecoles de Musiques, Ecoles bibliques (Instituts bibliques), Maison de la Bible...) ; le studio Neno la Uzima pour la $5{ }^{\text {ème }}$ CELPA, et l'Université Libre des Pays des Grands Lacs (ULPGL) ainsi que Ibra Radio, Radio Kahuzi, pour la $8^{\text {ème }}$ CEPAC et la $5^{\text {ème }}$ CELPA, l'Université Evangélique en Afrique pour la $8^{\text {ème }}$ CEPAC.

Le secteur Agro-pastoral : En dehors de certains champs pilotes et d'expérimentation qu'on trouve dans la ville de Bukavu, l'activité agropastorale est essentiellement pratiquée en milieu rural mais au compte des Eglises localisées en ville.

Soulignons que ce secteur sort de l'ordinaire, il emploie un personnel rural et urbain recruté par les Eglises urbaines. Le champ de Kashusha pour la $5^{\text {ème }}$ CELPA, plusieurs champs à Mulamba, à Katana et dans les paroisses rurales pour l'Eglise catholique romaine.

Le secteur paroissial : ce secteur regroupe les catégories professionnelles œuvrant directement ou indirectement dans les Eglises et/ou Paroisses. Il s'agit des agents tels que les Prêtres, les Pasteurs, les 
Evangélistes, les sacristains, les secrétaires, les caissiers, les comptables, les cuisiniers, domestiques, les gardiens/sentinelles, les techniciens (électriciens, chauffeurs, jardiniers) affectés dans les paroisses.

\section{Materiels et Methodes}

Le choix de la méthode structuro-fonctionnelle de Parsons (1973) s'est avéré indispensable dans cette recherche en vue d'identifier les sous-systèmes qui interagissent dans le contexte des emplois du secteur religieux afin de faciliter l'insertion professionnelle des chômeurs et la rémunération décente dans les services promus par les Eglises de Bukavu.

Comme démontré ici, cette étude a recouru à l'approche comparative pour établir les ressemblances et les différences entre les masses salariales accordées aux agents, le degré de satisfaction des agents au sein des services créés et gérés par Eglises de la Ville de Bukavu. La technique du questionnaire a été mise à contribution dans la collecte des données, alors que le logiciel SPSS 20 et le Khi2 interviennent dans le dépouillement et l'interprétation des données. La taille de l'échantillon de cet article est de 401 enquêtés (employés et responsables) sélectionnés dans 42 services de la $5^{\text {ème }}$ CELPA, $8^{\text {ème }}$ CEPAC et de l'Eglise catholique.

\section{Resultats}

\section{Les Eglises et la rémunération du personnel}

Sous cette section, cette étude passe en revue les modes de traitement appliqués aux employés dans les structures confessionnelles catholique et protestantes à Bukavu.

Pour Jean Paul II « Le rapport entre employeur et travailleur se résout sur la base du salaire, c'est-à-dire par la juste rémunération du travail accompli ». (Le Pape Jean Paul II, 1981). Cadin et al. sous-tendent que la question de la rémunération se trouve au cœur de la relation qui lie un employeur à ses salariés. La gestion de la rémunération permet à l'organisation d'attirer, de soutenir, d'encourager et de conserver une main d'œuvre efficace (Cadin et al., 2017).

La politique d'entreprise en matière de rémunération constitue donc l'ensemble de mécanismes mis en place pour récompenser et stimuler les travailleurs. Ce mécanisme se traduit par des revenus monétaires, des avantages ou des gains en nature.

Il est impérieux de distinguer à ce stade le salaire direct du salaire indirect. (Verret, M. (n.d.), p.55). Le premier (salaire direct) c'est le salaire en espèces perçu immédiatement de l'employeur par l'employé, en échange de son temps de travail, pour la couverture des besoins de reconstitution immédiate de sa force : besoins fractionnables, à renouvellement court, dont 
la périodicité règle d'ailleurs au départ celle du versement salarial (journée, semaine, quinzaine).

Le deuxième (salaire indirect) c'est le salaire en espèces ou en nature versé à l'ouvrier sur base viagère, c'est-à-dire à l'échelle de la vie toute entière, pour la couverture des frais d'entretien et de reproduction de sa force hors temps de travail. Salaire indirect, d'autres diront « disjoint » puisqu'il perd sa jonction immédiate à la dépense de travail, pour se retrouver joint à des événements aléatoires par rapport à celle-ci (maladie, accident) et extérieurs (naissances) ou postérieurs (retraite) à elle. (Hatzfeld, 1971) Salaire « social » dira-t-on encore, et à bien des titres. Social dans sa forme, puisque l'argent reçu ne l'est plus en fonction d'un contrat privé souscrit librement entre l'employé et l'employeur, mais d'un contrat social surajouté au premier, engageant, outre l'employé et l'employeur, une instance publique, Etat ou instances déléguées, exerçant contrainte légale.

Social dans sa forme, ce salaire l'est aussi en son contenu, puisqu'il garantit l'accès des salariés à des consommations sociales (services de soin, d'élevage, d'enseignement, de placement) ou collectives (transports, logements, équipements publics). (Tabard, 1974). Il l'est enfin dans sa fonction, puisque, sans mettre en cause le rapport d'emploi privé du salarié par le capital, il assure, par un prélèvement social direct (retraite) ou fiscale (charges sociales diverses).

Dans la pratique, on remarque plusieurs modes de détermination de salaire individuel, parmi lesquels, le salaire au rendement et le salaire d'efficience.

Outre ce mode, certaines entreprises prévoient le système de prime allouée à des groupes de travailleurs formant une certaine entité distincte, le SMIG (salaire minimum interprofessionnel garanti), le SMIL (salaire minimum interprofessionnel légal) et le panier de la ménagère.

Tous ces types de salaires ont été trouvés dans les services promus par les Eglises de Bukavu.

\section{Le salaire dans les structures professionnelles des Eglises de Bukavu}

Le salaire et la rémunération prêtent souvent à confusion. Pourtant, le premier fait partie intégrante de la deuxième. Cela veut dire que la rémunération est plus vaste que le salaire qui ne se limite qu'à l'appointement et au traitement d'activités.

La rémunération peut comprendre : a) le salaire ou traitement qui ne peut être inférieur au minimum légal, b) l'indemnité de vie chère. C'est une prime que l'on peut octroyer à l'employé pour la stabilisation de son niveau de vie, c) les sommes versées à titre de gratification qu'on appelle aussi paiement du $13^{\text {ème }}$ mois de l'année dans le sens d'offrir une fête de fin d'année, d) les sommes payées par l'employeur pendant l'incapacité de travail et 
pendant la période précédant la période et suivant l'accouchement. (Loi $\mathrm{n}^{\circ} 2002$ portant Code du Travail congolais, 2002)

Donnadieu (1997) quant à lui dresse une pyramide des rémunérations qui offre une vision schématique synthétique des différents modes de rémunération existant dans le cadre légal français. Elle est construite sur base du concept de rémunération globale qui prend en compte tous les avantages périphériques ainsi que des éléments de la rémunération différée (comme les systèmes d'intéressement) ou du salaire à long terme (comme l'épargne retraite constituée via le Plan d'Epargne pour la Retraite Collectif).

La pyramide de rémunération se décompose en quatre grands types de rémunérations :

- la rémunération principale: elle représente l'essentiel de la rémunération globale ;

- les périphériques légaux : ils ont pour caractéristique d'avoir fait l'objet d'une loi ou d'une ordonnance. Ils se traduisent, en fin de période de référence ou au bout d'un temps de blocage, par un versement d'argent qui vient compléter le salaire ;

- les avantages en nature: ce sont des éléments de rémunération variables et réversibles, souvent liés au statut de l'emploi occupé ;

- les périphériques statutaires: ils ont un caractère collectif, prédéterminé et rigide.

La rémunération est, de ce fait, la somme représentative de l'ensemble des gains, susceptibles d'être évalués en espèce et fixée d'un commun accord ou par des dispositions légales et réglementaires, qui sont dus en vertu d'un contrat du travail par un employeur à un travailleur.

A chaque travail, quelle que soit la position économique, sociale ou juridique de ceux qui le fournissent, doit correspondre une équitable rétribution. La rémunération dans les structures professionnelles des Eglises varie d'un service à un autre. Certains services à vocation sociale et de développement donnent une rémunération conséquente, alors que les autres services, surtout ceux attachés directement aux tâches de l'Eglise, allouent aux agents une rémunération relativement faible. Pour Allaire Yvan, la rémunération des dirigeants est en passe de devenir un enjeu politique, une cause de ressentiment social, une faille de gouvernance dans la plupart des sociétés développées. Quels que soient les arguments invoqués pour expliquer et justifier les sommes considérables versées aux dirigeants, la criante disparité des revenus au sein de la société et au sein même des entreprises fait de cet enjeu, au mieux, un cri de ralliement pour ceux qui veulent une société plus équitable, et au pire, une plateforme pour démagogues. Il est vrai que les disparités de revenus et de richesse sont inévitables dans une société méritocratique et que les retombées bénéfiques d'une économie de marché 
sont indissociables d'une certaine inégalité dans le partage de la richesse, mais ce n'est pas l'enjeu (Allaire, 2014).

Au-delà d'un certain seuil, la société devient inconfortable voire hostile, face à la fortune d'une minorité. Ce seuil tend à varier d'un pays à l'autre, d'une société à l'autre. Puis, la perception, l'impression que cette richesse n'a pas été honnêtement et franchement gagnée, qu'elle ne résulte pas d'une activité dont profite l'ensemble de la société exacerbe le malaise ou l'hostilité à l'encontre de la disparité des revenus et de la richesse. Dans le tableau ci-dessous, cette étude revient sur les masses salariales allouées aux agents dans les services des Eglises de Bukavu.

Tableau 1. Catégories de rémunération dans les structures professionnelles des Eglise

\begin{tabular}{|l|c|c|}
\hline Classes salariales en dollar & Fréquence & $\%$ \\
\hline 0 dollar & 9.00 & 2.24 \\
\hline 1 à 50 dollars & 23.00 & 5.73 \\
\hline 51 à 100 dollars & 89.00 & 22.19 \\
\hline 101 à 200 dollars & 111.00 & 27.68 \\
\hline 201 à 300 dollars & 89.00 & 22.19 \\
\hline 301 à 500 dollars & 60.00 & 14.96 \\
\hline 501 à 700 dollars & 13.00 & 3.24 \\
\hline 701 à 1000 dollars & 4.00 & 1.00 \\
\hline 1001 à 1500 dollars & 1.00 & 0.25 \\
\hline 1501 à 2000 dollars & 2.00 & 0.50 \\
\hline Total & 401.00 & 100.00 \\
\hline Rémunération moyenne & \multicolumn{2}{|c|}{215.73} \\
\hline Variance salariale/rémuné. & \multicolumn{2}{|c|}{0.152 .25} \\
\hline Ecart-type salarial & \multicolumn{2}{|c|}{} \\
\hline Coefficient de variation salariale & \\
\hline
\end{tabular}

Source : Enquête de terrain, Juin- septembre 2019

Les résultats présentés dans le tableau ci-dessus indiquent que le salaire moyen d'un employé dans les confessions religieuses est d'à peu-près 216 USD le mois. Le salarié le moins rémunéré gagne 0 dollar le mois. C'est un employé volontaire, il se rend disponible pour offrir ses services de manière bénévole sans attendre quelque chose de la part de son employeur. C'est une façon pour lui de communier avec son Eglise en lui rendant service gratuitement.

Le salarié le plus rémunéré gagne 2000 USD le mois. Cette catégorie professionnelle est constituée essentiellement des cadres gestionnaires des services promus par les Eglises. Le taux d'inégalité salariale mesuré par le coefficient de variation est de 47\%. Pendant que les Eglises sont considérées comme des lieux par excellence de justice sociale, l'on constate, à travers ces 
résultats, que l'écart est criant dans la rémunération des agents. Certains agents sont de loin, relativement bien payés, alors que d'autres ne touchent presque rien. Il est vrai que les services diffèrent du point de vue des investissements et des philosophies, mais les écarts discriminent un nombre important d'agents ; ce qui est source de démotivation totale et des lamentations.

Les salariés de grandes entreprises des confessions religieuses font parfois figure de «privilégiés » car leurs salaires dépassent souvent largement le salaire minimum en vigueur en R.D. Congo. Une minorité numérique s'accapare de gros moyens au détriment de la majorité qui ne garde que les miettes. C'est comme dans toute entreprise capitaliste, il y a une exploitation de la majorité par une minorité qui détient le pouvoir.

Presque la moitié du personnel employé dans les confessions religieuses gagne l'équivalent en Francs Congolais variant entre 51 et 200 dollars américains le mois. Cette rémunération est appréciée différemment par les confessions religieuses.

Tableau 2. Relation entre salaire et confession religieuse

\begin{tabular}{|c|c|c|c|c|c|c|c|c|}
\hline \multirow{3}{*}{$\begin{array}{l}\text { MONTANT DU } \\
\text { SALAIRE }\end{array}$} & & \multicolumn{5}{|c|}{ CONFESSION RELIGIEUSE } & \multicolumn{2}{|c|}{ Total } \\
\hline & \multicolumn{2}{|c|}{ 8ème CEPAC } & \multicolumn{4}{|c|}{ 5ème CELPA|CATHOLIQUE } & \multirow[b]{2}{*}{$\mathrm{F}$} & \multirow[b]{2}{*}{$\%$} \\
\hline & $\mathrm{F}$ & $\%$ & $\mathrm{~F}$ & $\%$ & $\mathrm{~F}$ & $\%$ & & \\
\hline 0 dollar & 0 & 0 & 2 & 2,29 & 7 & 3,80 & 9 & 2,24 \\
\hline 1 à 50 dollars & 4 & 3,07 & 3 & 3,44 & 16 & 8,69 & 23 & 5,73 \\
\hline 51 à 100 dollars & 34 & 26,15 & 19 & 21,83 & 36 & 19,56 & 89 & 22,19 \\
\hline 101 à 200 dollars & 29 & 22,30 & 41 & 47,12 & 41 & 22,28 & 111 & 27,68 \\
\hline 201 à 300 dollars & 41 & 31,53 & 12 & 13,79 & 36 & 19,56 & 89 & 22,19 \\
\hline 301 à 500 dollars & 12 & 9,23 & 7 & 8,04 & 41 & 22,28 & 60 & 14,96 \\
\hline 501 à 700 dollars & 4 & 3,07 & 2 & 2,29 & 7 & 3,80 & 13 & 3,24 \\
\hline 701 à 1000 dollars & 3 & 2,30 & 1 & 1,14 & 0 & 0 & 4 & 0,99 \\
\hline 1001 à 1500 dollars & 1 & 0,76 & 0 & 0 & 0 & 0 & 1 & 0,24 \\
\hline 1501 à 2000 dollars & 2 & 1,53 & 0 & 0 & 0 & 0 & 2 & 0,49 \\
\hline Total & 130 & 32,41 & 87 & 21,69 & 184 & 45,88 & 401 & 100 \\
\hline Moyenne & & & 25 & & 209 & & & \\
\hline
\end{tabular}

Source : Enquête de terrain, juillet 2019

Ce tableau renseigne que 9 travailleurs parmi lesquels 7 catholiques, soit $3,80 \%$ et 2 travailleurs de la $5^{\text {ème }}$ CELPA, soit 2,29\% touchent 0 dollar américain ou alors 0 franc congolais le mois ; 23 travailleurs, soit 5,73\% touchent entre 0 et 50 dollars le mois ; 89 agents parmi lesquels 34 de la 8 ème CEPAC, soit 26,15\%, 19 de la $5^{\text {ème }}$ CELPA, soit $21,83 \%$ et 36 catholiques, soit $19,56 \%$ reçoivent entre 51et 100 dollars le mois ; 111 employés, dont 41 de la $5^{\text {ème }}$ CELPA, soit 47,12\%, 41 catholiques, soit 22, $28 \%$ et 29 travailleurs, soit $22,30 \%$ ont un salaire variant entre 101 et 200 dollars le mois ; 89 agents encore parmi lesquels 41 de la $8^{\text {ème }}$ CEPAC, soit $31,53 \%, 12$ de la $5^{\text {ème }}$ 
CELPA, soit $13,79 \%$ et 36 catholiques, soit $19,56 \%$ bénéficient d'un salaire flottant entre 201 et 300 dollars ; 60 agents dont 12 de la $8^{\text {ème }}$ CEPAC, soit, $9,23 \% 7$ de la $5^{\text {ème }}$ CELPA, soit $8,04 \%$ et 41 catholiques, soit $22,28 \%$ sont rétribués par un montant oscillant entre 301 et 500 dollars ; 13 agents dont 4 de la $8^{\text {ème }}$ CEPAC, soit 3,07\%, 2 de la $5^{\text {ème }}$ CELPA, soit $2,29 \%$ et 7 catholiques, soit $3,80 \%$ obtiennent mensuellement un salaire fluctuant entre 501 et 700 dollars et enfin 7 agents, tous de la $5^{\text {ème }}$ CELPA et de la $8^{\text {ème }}$ CEPAC, reçoivent un salaire variant entre 701 et 2000 dollars. Aucun agent catholique parmi les enquêtés ne touche au-delà de 700 dollars.

Cette étude indique que la justice d'un système socio-économique et son fonctionnement harmonieux sont à apprécier d'après la manière dont on rémunère équitablement le travail humain dans ce système.

Tableau 3. Chi-Square Tests

\begin{tabular}{|l|l|l|l|}
\hline & Value & Df & Asymp. Sig. (2-sided) \\
\hline Pearson Chi-Square & 41.138 & 8 & .000 \\
\hline Likelihood Ratio & 46.352 & 8 & .000 \\
\hline Linear-by-Linear Association & 1.221 & 1 & .269 \\
\hline
\end{tabular}

Source : Enquête de terrain, 2019

On constate que le salaire diffère significativement selon les confessions religieuses. En effet, la moyenne salariale est de 225 dollars américains dans les confessions protestantes ( $5^{\text {ème }}$ CELPA et $8^{\text {ème }}$ CEPAC) contre 209 dans la confession catholique. Cette différence est significative, car indiquant que les catholiques rémunèrent moins leurs agents. En particulier, contrairement aux protestants, aucun travailleur ne touche pas plus de 700 dollars américains dans la confession catholique. La rémunération est prise au sérieux chez les protestants que chez les catholiques. Comme le démontrent les données de terrain, tout travail fourni dans le cadre de la structure professionnelle protestante est assorti de motivation ou mieux de la rémunération. Ceci n'est pas le cas chez certaines structures professionnelles catholiques qui, estiment que les chrétiens peuvent, surtout dans le cadre des travaux paroissiaux, rendre service de manière désintéressée au salaire.

L'appréciation salariale est significativement différente selon que l'enquêté est ou non catholique. Les catholiques sont les plus nombreux à être peu satisfaits.

\section{Autres avantages sociaux dans les Emplois des Eglises à Bukavu}

A côté du salaire direct, entrent en jeu diverses prestations sociales (salaire indirect) qui ont pour but d'assurer la vie et la santé des travailleurs et de leurs familles. Dans ce cadre, tout un système de droits particuliers se développe. Parmi ces droits, figure le droit à des lieux et des méthodes de travail qui ne portent pas préjudice à la santé physique des travailleurs et qui 
ne blessent pas leur intégrité morale. Les avantages (out comes) font fortunes diverses dans les confessions religieuses. S'ils sont reconnus et accordés à certains agents dans les structures professionnelles des Eglises à Bukavu, ils ne le sont dans d'autres.

Tableau 4. Relation confession religieuse et autres avantages que le salaire

\begin{tabular}{|l|l|l|l|}
\hline \multirow{2}{*}{ AVANTAGES HORS SALAIRE } & \multicolumn{2}{|c|}{ CONFESSION RELIGIEUSE } & \multirow{2}{*}{ Total } \\
\cline { 2 - 3 } & PROTESTANT & CATHOLIQUE & TAN \\
\hline OUI & $102(51.2 \%)$ & $104(51.4 \%)$ & 206 \\
\hline NON & $97(48.7 \%)$ & $98(48.5 \%)$ & 195 \\
\hline Total & 199 & 202 & 401 \\
\hline
\end{tabular}

Source : Enquête de terrain, août 2019

Le constat empirique nous montre que 206 enquêtés sont bénéficiaires d'autres avantages sociaux en plus des salaires qu'ils reçoivent régulièrement dans les emplois des Eglises de Bukavu. De ces 206 enquêtés, nous retrouvons 102 protestants, soit $51,2 \%$, contre 104 catholiques, soit 51,4\%, reconnaissant l'existence des avantages sociaux au sein de leurs services respectifs. Par contre, 195 enquêtés dont 97 protestants et 98 catholiques disent n'avoir jamais reçu les avantages sociaux dans leurs emplois.

Le contexte dans lequel évolue la structure professionnelle d'une Eglise influence énormément l'octroi ou pas des avantages sociaux aux agents. En fait, un service qui alloue des salaires modiques à ses agents, se heurte fréquemment aux contraintes de trouver les moyens financiers pour couvrir les avantages à leur octroyer. Cependant, certains chefs, parfois sans raison valable, empêchent leurs employeurs de jouir des avantages sociaux.

Tableau 5. Chi-Square Tests/confession religieuse et autres avantages que le salaire

\begin{tabular}{|l|c|c|c|}
\hline & Value & Df & Asymp. Sig. (2-sided) \\
\hline Pearson Chi-Square & 1.018 & 2 & .601 \\
\hline Likelihood Ratio & 1.404 & 2 & .496 \\
\hline Linear-by-Linear Association & .527 & 1 & .468 \\
\hline
\end{tabular}

Source : Logiciel SPSS 20, enquête de terrain, 2019

Les résultats indiquent que l'octroi des avantages aux salariés ne dépend pas de la confession. Ainsi, les catholiques se comportent de la même façon que les autres confessions. Il n'y a pas de particularité propre à une confession religieuse quant à l'octroi des prestations sociales.

Dans les différentes Eglises, les avantages sociaux (les congés, les gratifications dues aux performances, les allocations familiales, le transport) dépendent plutôt de capacités des services et des acteurs gestionnaires des emplois que des systèmes religieux. C'est dire que les Employeurs décident des avantages à accorder ou pas à leurs agents sans l'influence de l'Eglise. 
Le traitement, en cas de congé diffère significativement selon que l'on est chez les catholiques ou chez les protestants. En effet, les catholiques traitent moins bien leurs agents comparativement aux protestants. Le code du travail congolais prévoit qu'en cas d'absence injustifiée aucun salaire ne peut être dû, sauf pour les cas prévus par la réglementation ou la législation ou encore suivant les points de vue des parties intéressées. Toutefois, la rémunération est due lorsque le travailleur a été mis dans l'impossibilité de travailler pour l'employeur aussi bien que pour les jours fériés. (Article $72 \mathrm{du}$ code de travail de la R.D.C du 16 Octobre 2002) Il en résulte que plusieurs circonstances, notamment le deuil, la maternité, les congés, etc. peuvent amener l'employeur à payer son employé. Les modalités de paiement s'appuient tantôt sur la loi, tantôt sur les conventions collectives, tantôt encore sur les habitudes de l'entreprise. En cas d'absence de paiement, les agents procèdent par des cotisations spéciales au moyen des souscriptions à libérer lors de paiement des salaires mensuels. Face à une telle situation Durkheim (1960) a écrit que lorsque la société ne fournit pas un cadre stable, organisé et cohérent pour diviser le travail et fixer à chacun un rôle et des normes précises, l'anomie peut se produire.

\section{Le mode d'affectation de la rémunération aux besoins du travailleur}

La rémunération reçue par l'agent est affectée différemment selon ses priorités et le niveau du salaire qui lui est alloué à la fin du mois. Le tableau suivant nous permet de circonscrire les secteurs prioritaires dans lesquels les employés des Eglises affectent leur rémunération.

Tableau 6. Relation entre niveau de rémunération et mode d'affectation de celle-ci.

\begin{tabular}{|c|c|c|c|c|c|c|c|c|c|c|c|}
\hline \multirow[b]{2}{*}{$\begin{array}{l}\text { AFFECTATION DE L } \\
\text { A REMUNERATION }\end{array}$} & \multicolumn{9}{|c|}{ MONTANT DU SALAIRE EN DOLLARS AMERICAINS } & \multicolumn{2}{|c|}{ Total } \\
\hline & $\begin{array}{l}0- \\
50\end{array}$ & $\begin{array}{l}51- \\
100\end{array}$ & $\begin{array}{l}101- \\
200\end{array}$ & $\begin{array}{l}201- \\
300\end{array}$ & $\begin{array}{l}301- \\
500\end{array}$ & $\begin{array}{l}501- \\
700\end{array}$ & $\begin{array}{l}701- \\
1000 \\
\end{array}$ & $\begin{array}{l}1001- \\
1500\end{array}$ & $\begin{array}{l}1501- \\
2000\end{array}$ & $\mathbf{F}$ & $\%$ \\
\hline Nourriture & 21 & 40 & 28 & 16 & 21 & 2 & 2 & 1 & 0 & 131 & 32.6 \\
\hline Loyer & 3 & 6 & 12 & 19 & 18 & 0 & 0 & 0 & 0 & 58 & 14.4 \\
\hline Etudes des enfants & 0 & 3 & 19 & 14 & 2 & 0 & 0 & 0 & 0 & 38 & 9.4 \\
\hline Epargne & 0 & 14 & 6 & 0 & 1 & 0 & 0 & 0 & 0 & 21 & 5.2 \\
\hline Investissement & 0 & 3 & 4 & 18 & 2 & 0 & 0 & 0 & 0 & 27 & 6.7 \\
\hline $\begin{array}{l}\text { Les trois premiers cités } \\
\text { (études, Epargne et } \\
\text { investissement) }\end{array}$ & 8 & 23 & 42 & 22 & 16 & 11 & 2 & 0 & 2 & 126 & 31.4 \\
\hline Total & 32 & 89 & 111 & 89 & 60 & 13 & 4 & 1 & 2 & 401 & 100 \\
\hline
\end{tabular}

Source : Enquête de terrain, juillet 2019

Il ressort de ce tableau que 131 enquêtés, soit 32,66\%, affectent leur rémunération à l'achat de la nourriture ; 58 enquêtés, soit 14,48\%, l'affectent au paiement du loyer ; 38 enquêtés, soit 9,47\%, pour leur part l'affectent au paiement des frais scolaires et/ou académiques de leurs enfants ; 21 enquêtés, soit 5,23\%, affectent leur rémunération à l'épargne ; 27 enquêtés, soit 6,73\%, 
investissent leur rémunération dans des activités génératrices des revenus; 126 enquêtés enfin, soit $31,42 \%$, affectent leur salaire indistinctement dans le paiement de la nourriture, du loyer et de la scolarité des enfants.

De ce qui précède, l'on peut donc déduire que les employés des Eglises de Bukavu sont motivés au travail pour répondre prioritairement aux besoins physiologiques. La nourriture est la première préoccupation des agents des confessions religieuses à Bukavu. Elle est suivie par le désir de se payer le loyer et de supporter le coût de scolarité des enfants, alors que l'épargne n'est effectuée que rarement et par une faible proportion de nos enquêtés.

Les résultats indiquent que le mode d'affectation du salaire dépend significativement du niveau de celui-ci. En effet, lorsque le salaire est faible, il ne sert qu'à payer la nourriture et le loyer. Lorsqu'il est consistant, c'est alors que les salariés songent à la scolarisation de leurs enfants, en plus de l'épargne et de l'investissement. Du coup, la relation entre le salaire reçu et son affectation par l'employé conduit à deux dimensions de conséquences qui sont unies entre elles et se complètent sur différents points :

$\mathrm{Du}$ point de vue économique, les résultats rejoignent la thèse selon laquelle, la propension marginale à consommer diminue avec l'augmentation du revenu. Les ménages pauvres consomment presque la totalité de leurs revenus. Les résultats indiquent que les salariés des Eglises enquêtés reçoivent un salaire de subsistance car très peu seulement d'entre eux songent à l'épargne et à l'investissement.

Du point de vue social, les masses salariales allouées aux travailleurs déterminent leur niveau de vie et de satisfaction au sein du service. La satisfaction des besoins prioritaires des agents constitue en même temps le facteur de motivation d'un grand nombre du personnel.

\section{Discution des Resultats}

Comme le montre l'étude, le salaire moyen d'un employé des structures professionnelles des Eglises de Bukavu est faible, soit 216 dollars. Contrairement à l'affirmation de Weber (2000) qui voit dans la carrière du travailleur puritain un appel, «une vocation» et que la spécificité du puritanisme est précisément la soumission de « l'appât du gain » à une éthique rationnelle, les travailleurs des Eglises de Bukavu qui ne touchent pas un salaire proportionnel à leur travail disent que leurs prestations doivent être considérées comme un apostolat ou mieux un acte de charité à l'égard des enfants de Dieu.

Il ressort que la satisfaction de cette catégorie d'agents n'est pas directement influencée par le stimulant financier, mais par le contexte des relations humaines qui prévaut dans le service. Plusieurs agents sont ainsi convaincus que travailler dans une structure professionnelle, quels que soient les salaires qu'ils reçoivent, est une garantie pour garder leur croyance 
religieuse. Sachant que le travailleur est parfois incité à répondre à un symbole ou un stimulis de salaire au rendement, au regard du salaire moyen des employés des Eglises, l'on pourrait se demander leur niveau de motivation dans l'exécution des tâches qui leur sont dévolues.

Cependant, dans la mesure où le travail a pour finalité l'échange marchand, l'individu n'est plus maître de déterminer lui-même le niveau de ses besoins et celui de l'effort qu'il est prêt à fournir. La rationalité économique recule toujours la limite de la nécessité : elle exclut la notion de suffisant : «L'établissement d'une norme du suffisant est incompatible en raison de l'autolimitation des besoins et de l'effort consenti qu'elle implique- avec la recherche du rendement maximum qui constitue l'essence de la rationalité et de la rationalisation économiques ». (Gorz, 1992)

En outre, cette recherche révèle que le taux de satisfaction du salaire par les agents, dans l'ensemble, est déplorable. Pour Gorz (1988), « la catégorie du suffisant n'est pas une catégorie économique : c'est une catégorie culturelle ou existentielle. Ce qui est suffisant est ce qu'il y a de mieux ».

Herzberg (1959) dégage cinq facteurs de satisfaction à savoir l'accomplissement, la reconnaissance de l'accomplissement, le travail luimême, la responsabilité et la progression sociale. Les facteurs de mécontentement touchent plus à l'environnement qu'aux relations entre les hommes: la politique de l'administration, la personne du supérieur, la rémunération, les relations avec les collègues, les conditions de travail. Ce sont les facteurs d'hygiène.

Le traitement du personnel dans les structures professionnelles des Eglises crée de l'insatisfaction chez certains travailleurs. Il est vrai que la satisfaction n'agit pas forcément dans le sens d'une motivation individuelle favorable à l'organisation, mais elle peut, à un certain niveau, en déterminer la productivité. Pour atteindre une satisfaction qui marie la productivité de l'entreprise, la théorie de besoins et de motivations souligne l'importance des réactions stratégiques.

Selon Maslow (1943), le salaire est vital. Les travailleurs attendent de l'entreprise une rémunération qui leur permette de vivre et de se développer avec leurs familles.

Or comme démontré dans cet article, les structures professionnelles religieuses allouent un salaire ne permettant pas à leurs bénéficiaires de répondre aux besoins alimentaires de leurs familles respectives. L'homme exprime plusieurs besoins à la fois et quand un besoin est satisfait, un autre surgit et se substitue au précédent.

Dans sa théorie de deux facteurs, Herzberg (1959) montre que la satisfaction au travail fonctionne séparément de l'insatisfaction au travail. Le contraire de la satisfaction étant le manque de satisfaction. 
Les conséquences sont donc qu'on peut être à la fois satisfait et insatisfait dans son travail. Selon le même auteur, la satisfaction porte sur nos responsabilités et réalisations (le haut de la pyramide de Maslow) et l'insatisfaction des besoins (bas de la pyramide de Maslow).

Choisir une récompense appropriée est souvent assez difficile car certains hommes sont motivés par les salaires ; pour d'autres, par un voyage et pour d'autres encore un beau bureau ... La récompense doit être généreuse, équitable, attirante comme le souligne Victor Vroom (1995) dans sa théorie des attentes. Cette théorie contrairement à celles de Maslow ou Herzberg, ne se focalise pas sur les besoins des personnes, mais relie la motivation d'un individu à ses attentes et les chances qu'il possède de les atteindre. (Roussel, 2015, (n.d.))

\section{Conclusion}

La rémunération joue un rôle capital dans l'amélioration des performances de production. Elle constitue un facteur motivationnel qui pousse l'homme à s'engager avec courage et détermination dans le processus de production. Mais, bien qu'elle soit capitale, la rémunération s'affiche souvent en double face paradoxale. Elle représente en même temps un facteur d'équilibre social pour les salariés et un coût pour l'Entreprise. D'où la nécessité pour un gestionnaire des ressources humaines de concilier les deux faces.

Cet article essaye de comprendre trois dimensions de la rémunération dans les secteurs professionnels promus par les Eglises dans la Ville de Bukavu.

Il s'est agi d'analyser les facteurs, le taux de salaires et le degré de satisfaction des agents en tenant compte de leur profil et participation au fonctionnement harmonieux des entreprises qui les utilisent.

A l'issue des investigations, il est ressorti que : la rémunération dans les structures professionnelles des Eglises varie et prend de l'ampleur d'un service à un autre. Certains services à vocation sociale et de développement donnent une rémunération conséquente, alors que les autres services, surtout ceux attachés directement aux tâches de l'Eglise, allouent aux agents une rémunération relativement faible. Le salaire moyen d'un employé dans les confessions religieuses est d'à peu-près 216 dollars américains le mois. Le salarié le moins rémunéré gagne 0 dollar le mois et le salarié le plus rémunéré gagne 2000 dollars américains le mois. Le taux d'inégalité salariale mesuré par le coefficient de variation est de $47 \%$.

Pendant que les Eglises sont considérées comme des lieux par excellence de justice sociale, l'on constate, à travers cet article que l'écart est criant dans la rémunération de leurs agents. Il est vrai que les services diffèrent du point de vue des investissements et des philosophies, mais les écarts 
discriminent un nombre important d'agents en les mettant dans des lamentations.

L'appréciation salariale est significativement différente selon que l'enquêté est ou non catholique. Les catholiques sont les plus nombreux à être peu satisfaits. Par ailleurs, le taux de satisfaction, dans l'ensemble, est déplorable.

\section{References:}

1. Allaire, Y. (2014). Les inégalités économiques et la rémunération des dirigeants d'entreprises, Paris, éd. Les affaires.

2. AGRH (2016). www.reims-ms.fr/agrf consulté le 12 mai.

3. Cadin, L., Geurin, F., \& Pigeyre, F. (2007). Gestion des ressources humaines : pratique et éléments de théories, Paris, Dunod.

4. Cishunguluka, K. A. (2017). Entreprise coopérative d'épargne et de crédit : Lutte contre la pauvreté et pour la souveraineté sociale locale à Bukavu, Thèse de Doctorat, Université de Kisangani, Département de Sociologie, 119.

5. Code de Travail de la R.D. Congo, Loi N ${ }^{\circ}$ 15/2002 du 16 Octobre 2002

6. Donnadieu, G. (1997). Du salaire à la rétribution, Paris, Editions liaisons, 142.

7. Duquesne, J-J. (n.d.).Les différentes catégories de la rémunération, www.legifrance.gouv.fr, consulté le 8 novembre 2017 à 8 h 27min.

8. Durkheim, E. (1960). Les formes élémentaires de la vie religieuse. Le système totémique en Australie, Paris, Quadrige, PUF, 201.

9. Friedmann, G. \& Naville, P. (1972). Traité de sociologie du travail, $3^{\text {ème }}$ édition, Paris, Armand Colin, 132.

10. Guillot-Soulez, C. (2010). Gestion des Ressources Humaines, 3 ème édition, Paris, Gualino, 118-120

11. Gorz, A. (1992). «L'écologie politique entre expertocratie et autolimitation », Actuel Marx, «L'écologie, ce matérialisme historique », $\mathrm{n}^{\circ} 12,22$.

12. Gorz, A. (1988). Métamorphoses du travail, Quête du sens, Critique de la raison économique, Paris, Galilée, 142.

13. Hatzfeld, M. (1971). Du paupérisme à la sécurité sociale 1876-1947, Paris, Armand Colin, 47.

14. Herzberg, F., Mausner, B., \& BB. (1959). Motivation to work, New York, John Weley, 17.

15. Http://www.zenit.org/article-12646 ?1=french consulté le 12 mars 2016

16. Lecuyer, P., Boudon, R., Besnard, P. \& Mohamed, C. (2003). Dictionnaire de sociologie, Larousse, Paris, VUEF, 720. 
17. Maslow, A. (1943). «A theory of human motivation», The psychological Review, Vol.50, N4, 370-396

18. Mokakando, A. B. (2011-2012). Etude des principes des ressources humaines et attentes dans l'administration publique congolaise et les entreprises privées de Kisangani, Thèse de Doctorat, Université de Kisangani, Département de Psychologie, 99.

19. Pape Jean Paul II (1981). Laborem exercens, Kinshasa, Ed. Saint Paul Afrique, 32.

20. Parsons, T. (1973). Sociétés. Essai sur leur évolution comparée (1996), Paris, Dunod, 36

21. Pilo, K. P. \& Rugambwa, S. J. (2005) « Analyse critique des aspects historiques et écologiques de la ville de Bukavu et perspectives d'avenir », in Ujuvi, numero16, CRI, 11-39.

22. Roussel, P. (2015). La motivation au travail, alain. Battandier.free.fr spip consulté le 8 mars.

23. Tabard, N. (1974). Besoins et aspirations des familles et des jeunes, Paris, CREDOC/UNCAF, 17.

24. Urech, P. (2017). La différence entre la rémunération et le salaire, https://www.petite-entreprise.net consulté le 7 novembre.

25. Verret, M.(n.d.). Le travail ouvrier, Paris, Armand Colin, 55.

26. Vroom, V. (1995). Work and Motivation, https.//www.worldcat.org consulté le 11 avril 2018

27. Weber, M. (2000). L'éthique protestante et l'esprit du capitalisme, Paris, Flammarion, champs, 57.

28. Werther, W.B. et al. (1990). La gestion des ressources humaines, $2^{\text {ème }}$ édition, Québec, Canada, 436. 